\title{
Tackling the challenges with poorly soluble drugs
}

\author{
Keywords HPMC, excipients, crystallinity, temperature, melting \\ point
}

\section{Introduction}

A significantly large percentage of new chemical entities is insoluble and belongs to BCS Class II and Class IV. ${ }^{1}$ Insolubility is due to high crystallinity and melting temperature and partitioning coefficient in an organic phase, which can be described by the Equation $1 .^{2}$

$$
\log S_{w}(M / L)=0.8-\log K_{o w}-0.01(M P-25) \quad \text { Equation } 1
$$

Where, $\mathrm{S}_{\mathrm{w}}$ is the aqueous solubility of the drug expressed in $\mathrm{mol} / \mathrm{L}, \mathrm{K}_{\mathrm{ow}}$ is the partition coefficient in 1-octanol and water, MP is the melting point in ${ }^{\circ} \mathrm{C}$.

The physical modifications of molecules including milling/ micronization or $\mathrm{pH} / \mathrm{salt}$ formation often show limited success in terms of dissolution but the non-traditional formulation approaches can lead to a significant increase in dissolution and solubility particularly required for drugs applied in medium to higher dosages. ${ }^{3}$ Such dosages' requirements go along with the necessity to evaluate other formulation technologies for enhancing solubilization. ${ }^{4}$ For instance, polymer based solid amorphous dispersions and surfactant based liquid self-emulsifying systems are alternative approaches to address these solubilization challenges. With the availability of innovative excipients, performance and robustness of drug delivery systems can be enhanced and offer more possibilities than ever before. For instance, Kaletra ${ }^{\circledR}$ marks the earlier tradition of transfer from lipid based emulsifying soft gel capsules (in 2000) to polymer based solid amorphous dispersion tablets (in 2005).

This review examines the importance of excipients in solubilization and their roles in preventing crystallization and maintaining the supersaturation in enhancing the bioavailability of drug molecules.

\section{Self-emulsifying/micro-emulsifying drug delivery systems (SEDDS/SMEDDS)}

Surfactant/lipid based self-emulsifying/micro-emulsifying drug delivery systems (SEDDS/SMEDDS) have been used effectively for many of poorly soluble lipophilic molecules with $2<\log \mathrm{P}>8$. Excipients for liquid emulsifying systems have tendencies to simultaneously self-assemble or aggregate in aqueous solutions, allowing the drug molecules to partition between the aqueous phase and the hydrophobic interior and maintain the drugs in solution for an extended period for absorption through the gastrointestinal (GI) tract. ${ }^{6}$ These properties of lipid based excipients stem from hydrophilic and lipophilic characteristics of molecules, which are identified by hydrophilic lipophilic balance (HLB) values. The molecules with higher HLB values are more hydrophilic and vice versa and spontaneously aggregate into micelles. The examples of surfactant/ lipid based solubilizers include Kolliphor $^{\mathbb{R}}$ EL (HLB 12-14), RH40 (HLB 14-16), HS15 (HLB 16), TPGS (HLB 16), PS80 (HLB 16), P188 (HLB 29), P407 (HLB 23) among others. These surfactants possessing also lipophilic characteristics, will encapsulate the hydrophobic or lipophilic drug molecules in the micelle cores. ${ }^{7}$
Volume I Issue I - 2015

\author{
Shaukat Ali, Karl Kolter \\ 'BASF Corporation, USA \\ ${ }^{2}$ BASF SE, Germany
}

Correspondence: Shaukat Ali, BASF corporation, 100 park avenue, Florham park, NJ 07932, USA, Tel + 1003420050 , Email shaukat.ali@basf.com

Received: May 15, 2015 | Published: September 02, 2015

A self-emulsifying process results in a spontaneous distribution of drugs into the micellar core upon agitation. Thus, the presence of water is critical during emulsification and maintenance of drug in solution. Figure 1 illustrates the spontaneous formation of small droplets from a pre-concentrate (cocktail) comprising drug and surfactants based lipids. ${ }^{8}$ Upon dilution, the pre-concentrate is dispersed into an emulsion and further micro-emulsified into smaller droplets as the process continues before being absorbed in GI tract. Faster mixing and dispersing abilities of the active within the surfactants depend on type and amounts of surfactants (higher HLB values) and/or cosurfactants (lower HLB values). Evidently, the higher the percentages of solubilizers, the faster the dispersing process and the smaller the particle size are, and hence, the better the absorption in GI tract gets (Figure 1). ${ }^{9}$

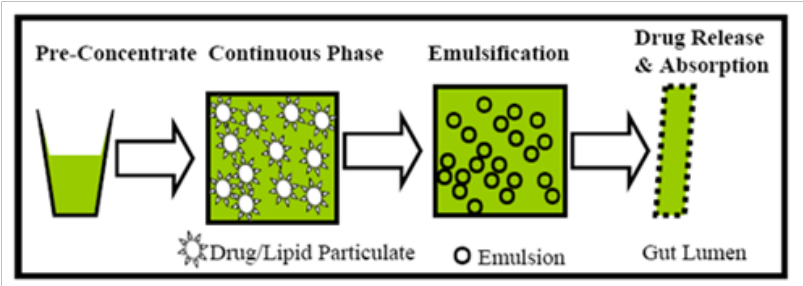

Figure I A self-emulsifying process from the pre-concentrate (reproduced with permission from). ${ }^{8}$

Such formulations provide advantages over tablet formulations due to ease of scale up and manufacturing process, and faster development including drug launch. ${ }^{10}$ Example of marketed drugs in surfactant/lipid

based self-emulsifying systems approved are Agenerase $^{\circledR}$ (Amprenavir); Norvir ${ }^{\circledR}$ (Ritonavir); Kaletra ${ }^{\circledR}$

(Ritonavir/Lopinavir); Fortovase ${ }^{\circledR} \quad$ (Saquinavir); Aptivus ${ }^{\circledR}$ (Tipranavir); Neoral ${ }^{\circledR}$ (Cyclosporine); Sandimmune ${ }^{\circledR}$ (Cyclosporine); Gengraf $^{\mathbb{B}}$ (Cyclosporine).

\section{Amorphous solid dispersions}

Amorphous solid dispersion (ASD) offers an alternative approach to increase the solubility and enhance bioavailability, especially, for those with high crystalline and high melting characteristics. Converting highly insoluble and stable crystalline API to highly soluble dispersion also brings challenges due to instability of the 
higher energy amorphous state. ${ }^{11}$ Polymers play an important role in stabilization of amorphous APIs. Therefore, finding the polymers with desired properties such as good solubilization, high thermal stability, low/no hygroscopicity, no toxicity are important in development of an ASD dosage. Formulation technologies such as hot melt extrusion (HME), spray drying, co-precipitation, Kinetisol ${ }^{\circledR}$, melt granulation, electro-spinning and electro-spraying processes have been used, some more extensively than others. ${ }^{12-15}$ Amongst all, the HME is widely used because it is a solvent free process, amenable to various downstream processing steps, and the availability of suitable pharmaceutical polymers and solubilizers makes it viable to improve efficacy and safety of the dosage forms. This review highlights the key attributes of HME process, and the polymers suited for this technology.
Figure 2 illustrates the HME process in which a polymer when heated, typically at approx. $50^{\circ} \mathrm{C}$ above its glass transition temperature $\left(\mathrm{T}_{\mathrm{g}}\right)$, melts and acts as a solvent, dissolving the crystalline API. The entire process (melting of polymer and dissolving of API) takes about 2 minutes or less, and the crystalline drug is fully converted to an amorphous dispersion or solid solution. ${ }^{16}$ Increasing amounts of drugs could lead to precipitation and phase separation in the polymeric matrix, which is basically characterized by the melting temperature of API and the individual $\mathrm{T}_{\mathrm{gs}}$ of drug and polymer. The glassy extrudate with drug molecules completely dissolved in the polymer can be further processed into tablets or cut into desired pellets.

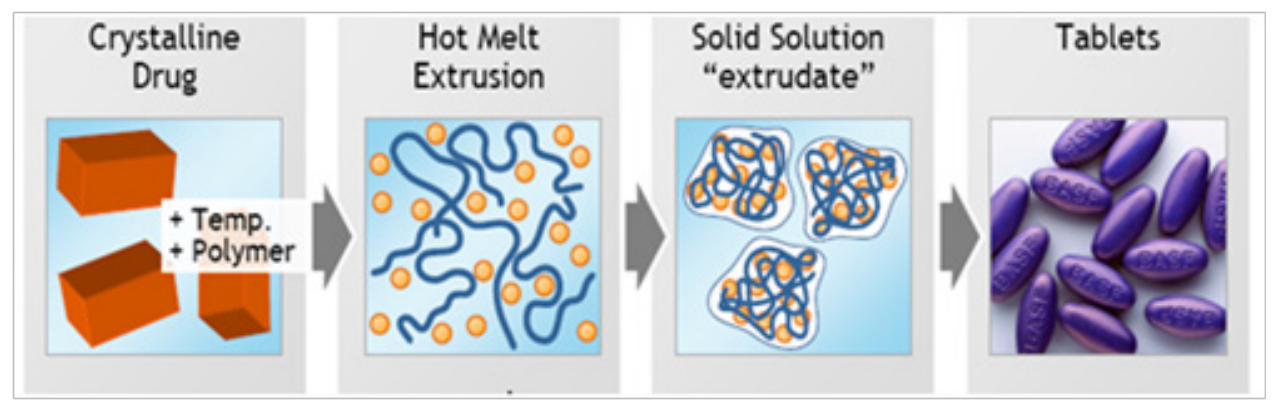

Figure 2 Illustration of melting and mixing of a crystalline API in polymer by hot melt extrusion (reproduced with permission from). ${ }^{8}$

Table I $\mathrm{T}_{\mathrm{g}}$ and $\mathrm{T}_{\text {deg }}$ of different polymers*

\begin{tabular}{|c|c|c|c|c|c|c|c|c|c|}
\hline $\begin{array}{l}\text { Temp } \\
\left({ }^{\circ} \mathrm{C}\right)\end{array}$ & Kollidon VA64 & I Soluplus & $\begin{array}{l}\text { Kollidon } \\
\text { K-I } 2\end{array}$ & $\begin{array}{l}\text { Kollidon } \\
\text { K-I7 }\end{array}$ & $\begin{array}{l}\text { Kollidon } \\
\text { K-30 }\end{array}$ & $\begin{array}{l}\text { Kollidon } \\
\text { K-90 }\end{array}$ & $\begin{array}{l}\text { Kollidon } \\
\text { SR }\end{array}$ & $\begin{array}{l}\text { Kollicoat } \\
\text { MAE I 00P }\end{array}$ & Kollicoat IR \\
\hline $\mathrm{T}_{\mathrm{g}}$ & 101 & 70 & 90 & 138 & 149 & 156 & 39,152 & 114 & $45,208 \ddagger$ \\
\hline$T_{\text {deg }}$ & 230 & 250 & 138 & 175 & 175 & 200 & 210 & 150 & 200 \\
\hline
\end{tabular}

$\ddagger$ indicates the melting point of the polymer.

* $\mathrm{T}_{\mathrm{g}}$ was determined by Differential Scanning Calorimetry (DSC) and $\mathrm{T}_{\text {deg }}$ was determined by Thermo gravimetric analysis (TGA).

Table 1 lists a number of polymers and their $\mathrm{T}_{\mathrm{g}}$ and $\mathrm{T}_{\mathrm{deg}}$. These values are crucial for understanding the processing conditions of APIs with polymers for extrusion. Interestingly, nearly all polymers are thermally stable at fairly higher temperatures and will be suited for HME (Table 1). ${ }^{17,18}$

Intermolecular interactions with API and polymer play an important role in stabilization of ASD. The stability also depends on the molecular weight and chain lengths of polymers. For instance, PVP K-30 with longer chains will have much greater entanglement and stronger interactions with drug molecules as compared to PVP K-12. In addition, the polymers with amide functional moieties will have better interactions than those lacking the same such as hydroxypropyl cellulose (HPC), hydroxypropyl methyl cellulose (HPMC), or hydroxypropyl methyl cellulose acetyl succinate (HPMCAS). In contrast, polymers such as $\mathrm{PVP}\left(\right.$ Kollidon $\left.^{\circledR}\right)$, copovidone (Kollidon ${ }^{\circledR}$ VA64, and Soluplus ${ }^{\circledR}$ ) will have much stronger intermolecular interactions with APIs due to inherent binding sites $(\mathrm{C}=\mathrm{O})$ distributed throughout the polymeric chains, and their complexing abilities via hydrogen bonding between API and polymer, as shown in Figure
3. ${ }^{8}$ These interactions are important in preventing nucleation, crystallization, and maintaining the super saturation of drugs in aqueous and bio-relevant dissolution media. ${ }^{19}$ The disruptions of $\mathrm{H}$-bonds in ASD could have implications on enhancing the dissolution. Hughey et al. ${ }^{20}$ have demonstrated that the addition of inorganic salt enhances the dissolution by competing with the hydrogen bonding between API and Soluplus ${ }^{\circledR} .{ }^{20}$ In a recent study, Soluplus ${ }^{\circledR}$ significantly increased the solubility of itraconazole and tremendously enhanced the bioavailability in beagle dogs as compared to crystalline drug in a physical mixture (26-fold). ${ }^{21,22}$

Example of amorphous solid dispersion based on marketed drugs include Kaletra ${ }^{\circledR}$ (Ritonavir/Lopinavir); Norvir ${ }^{\circledR}$ (Ritonavir); Viekira Pak ${ }^{\mathrm{TM}}$ (Dasabuvir and Ombitasvir and Paritaprevir and Ritonavir); Belsomra $^{\circledR}$ (Suvorexant); Noxafil ${ }^{\circledR}$ (Posaconazole); Sporanox ${ }^{\circledR}$ (Itraconazole); Intelence $^{\circledR}$ (Etravirine); Prograf $^{\circledR}$ (Tacrolimus); Crestor $^{\circledR} \quad$ (Rosuvastatin); Gris-PEG ${ }^{\circledR} \quad$ (Griseofulvin); Cesamet $^{\circledR}$ (Nabilone); Solufen ${ }^{\circledR}$ (Ibuprofen); Zelboraf ${ }^{\circledR}$ (Vemurafenib); Incivek $^{\circledR}$ (Telaprevir) 


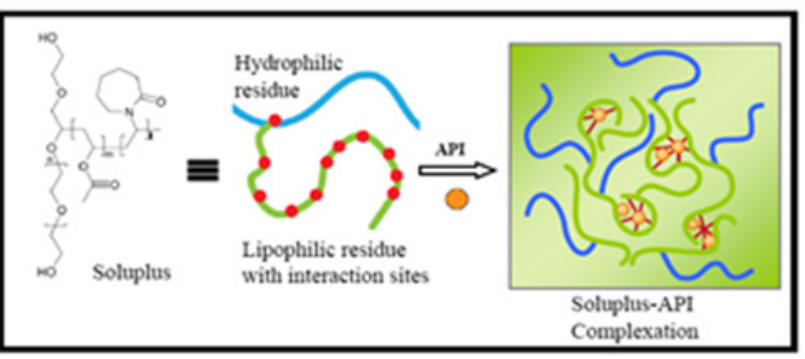

Figure 3 Complexing ability of Soluplus ${ }^{\circledR}$ (MW I I8,000) with an API.

\section{Conclusion}

In summary, this article highlights the importance of commonly used surfactant/lipid based self-emulsifying delivery systems (SEDDS/SMEDDS), and polymer based amorphous solid dispersions to meet the challenges with poorly soluble NCEs. These solubilization technologies could equally be important to generic drugs to overcome the challenges with existing process and formulation limitations for designing the better pills to avoid patent infringements. The availability of a wide range of pharmaceutically accepted polymers and solubilizers opens doors too many poorly soluble drugs which otherwise will not find their ways into drug development. Soluplus ${ }^{\circledR}$, Kollidon $^{\circledR}$ VA64, HPMCAS are among only few polymers that offer unique opportunities for many poorly soluble compounds. As the numbers of Class II and Class IV compounds continue to rise, there will be a growing need for designing better solubilizers. However, new chemical entities in excipients pose a certain challenge from a regulatory point of view, since such compounds have not been approved before. From the perspective of a pharmaceutical company this is associated with an increased risk of getting the approval sooner than later. All parties involved in drug development and approval have to find a way to overcome this hurdle, which for instance, could be due to direct approval of an excipient for a certain route of administration and not associated with a drug product in general.

\section{Acknowledgments}

None.

\section{Conflicts of interest}

The author declares that there is no conflict of interest.

\section{References}

1. Giliyar C, Fikstad D, Tyavanagimatt S. Challenges and opportunities in oral delivery of poorly water-soluble drugs. Drug Delivery Technol. 2006;6:57-63

2. Yalkowsky SH. Solubility and Solubilization in Aqueous Media. Oxford University Press. USA. 1999:67.

3. Hauss DJ. Oral lipid based formulations: enhancing the bioavailability of poorly water soluble drugs. London, UK: Informa Healthcare; 2007:138

4. Breitenbach J. Melt extrusion: from process to drug delivery technology. Eur J Pharm Biopharm. 2002;54(2):107-117.
5. Gathe JC, Lipman BA, Mayberry C, et al. Tolerability and therapy preference of lopinavir/ritonavir (Kaletra) soft gel Capsules and tablets as single agent in a cohort of HIV positive adult patients (IMANI-2), $8^{\text {th }}$ International Congress on Drug Therapy in HIV Infection. Glasgow. 2006:62.

6. Müllertz A, Ogbonna A, Ren S, et al. New perspectives on lipid and surfactant based drug delivery systems for oral delivery of poorly soluble drugs. J Pharm Pharmacol. 2010;62(11):1622-1636.

7. Reintjes T. Solubility enhancement with BASF pharma polymers: Solubilizer compendium. BASF SE Pharma Ingredients \& Services. Germany. 2011.

8. Ali S, Kolter K. Challenges and opportunities in oral formulation development. Am Pharm Rev. 2012:s14-s21.

9. de Smidt PC, Campanero MA, Trocóniz IF. Intestinal absorption of penclomedine from lipid vehicles in the conscious rat: contribution of emulsification versus digestibility. Int J Pharm. 2004;270(1-2):109-118.

10. Benza H, Munyendo W LL. A review of progress and challenges in soft gelatin capsules formulations for oral administration. Int J Pharm Sci Rev Res. 2011;10:20-26.

11. Padden BE, Miller JM, Robbins T, et al. Amorphous solid dispersions as enabling formulation for discovery and early development. Am Pharma Rev. 2011:66-73.

12. DiNunzio JC, Brough C, Miller DA, et al. Applications of KinetiSol ${ }^{\circledR}$ dispersing for the production of plasticizer free amorphous solid dispersions. Eur J Pharm Sci. 2010;40(3):179-187.

13. Moneghini M, De Zordi N, Solinas D, et al. Characterization of solid dispersions of itraconazole and vitamin E TPGS prepared by microwave technology. Future Med Chem. 2010;2(2):237-246.

14. Ali S, Langley N, Djuric D, et al. Electrospinning for Solid Dispersions of Poorly Soluble Drugs. 2011.

15. Nagy ZK, Balogh A, Vajna B, et al. Comparison of electrospun and extruded Soluplus $^{\circledR}$-based solid dosage forms of improved dissolution. J Pharm Sci p. 2011:1-14.

16. Smithey D, Fennewald J, Gautschi J, et al. Evaluation of the Polymer Soluplus ${ }^{\circledR}$ for Spray-Dried Dispersions of Poorly Soluble Compounds. 2010

17. Kolter K, Karl M, Gryczke A. Hot melt extrusion with BASF pharma polymers: Extrusion Compendium. BASF the chemical company. Germany. 2012:192.

18. Ali S, Langley N, Djuric D, et al. Soluplus- a novel polymer for hot melt extrusion. Tablets \& Capsules. 2010.

19. Tsinman O, Tsinman K, Ali S. Soluplus ${ }^{\circledR}$ : An understanding of super saturation from amorphous solid dispersions. Drug Dev Delivery. 2015.

20. Hughey JR, Keen JM, Miller DA, et al. The use of inorganic salts to improve the dissolution characteristics of tablets containing Soluplus ${ }^{\mathbb{B}}$ based solid dispersions. Eur J Pharm Sci. 2013;48(4-5):758-766.

21. Hardung H, Djuric D, Ali S, et al. Combining HME and Solubilization:Soluplus ${ }^{\circledR}$-The Solid Solution. Drug Del Technol. 2010;10(3).

22. Linn M, Collnot EM, Djuric D, et al. Soluplus ${ }^{\circledR}$ as an effective absorption enhancer of poorly soluble drugs in vitro and in vivo. Eur J Pharm Sci. 2012;45(3):336-343. 